\title{
OXÍGENO HIPERBÁRICO EN EL MANEJO DE RADIONECROSIS DEL TALLO CEREBRAL EN UNA PACIENTE CON MÉDULOBLASTOMA
}

\author{
Eduardo DuRÁn Pinilla, MD, \\ Neurocirujano, Jefe unidad de Medicina Hiperbárica, Hospital Militar Central. \\ GERMÁN VALDERRAMA SERRANO, MD, \\ Cirujano, Medicina Hiperbárica, Hospital Militar Central. \\ Alejandro Buitrago Salinas. MD, \\ Cirujano, Medicina Hiperbárica, Hospital Militar Central. \\ Unidad de Medicina Hiperbárica, Hospital Militar Central, Bogotá D.C., Colombia. \\ CONTACTO PARA INFORMACIÓN Y CORRESPONDENCIA \\ Dr. Eduardo Durán Pinilla \\ Hospital Militar Central. Transversal 3 $3^{a} N^{o} 49$ - 00, Bogotá D.C. \\ eduardoduránpinilla@yahoo.com
}

PROCEDENCIA DE APOYO O FINANCIACIÓN

No se recibió ningún tipo de aporte económico o en especie con esta finalidad. Los recursos para la atención y prestación del servicio provienen de lo dispuesto por el Plan Obligatorio de Salud de Colombia que de manera rutinaria se utiliza en la atención de cualquier paciente.

TÍTULO ABREVIADO:

RADIONECROSIS DEL TALLO CEREBRAL

\begin{abstract}
Resumen
La radionecrosis del tallo cerebral es una de las complicaciones de la radioterapia en pacientes que han recibido tratamiento para un tumor, y aunque afortunadamente no es lo más frecuente, sus consecuencias pueden ser devastadoras, pues la importancia del tejido comprometido hace que se generen lesiones que pueden producir secuelas altamente incapacitantes y hasta la muerte del paciente como en efecto sucedió en el presente caso. Según la FDA (Food and Drug Administration) el Oxígeno Hiperbárico $(\mathrm{OHB})$ se encuentra indicado en la radionecrosis ósea y radionecrosis de tejidos blandos, incluyendo en esta última al sistema nervioso central. El caso reportado es el de una paciente de 28 años de edad, con un meduloblastoma de fosa posterior que fue tratado con cirugía y químio-radioterapia. 21 meses después del tratamiento inicial presenta síntomas que sugieren una lesión en el tallo cerebral, por lo que nuevamente consulta y posterior a estudios imagenológicos se logra determinar que se trata de una lesión del tallo cerebral por radionecrosis. En julio de 2011 la paciente ya presentaba disartria, bradilalia, lateropulsión izquierda, diplopía, cefalea, marcha atáxica y espasticidad simétrica con predominio del hemicuerpo izquierdo. Al momento de iniciar el tratamiento con OHB (septiembre de 2011), se encontró cuadriparesia no funcional. Se realizaron 6 sesiones con $\mathrm{OHB}$, tratamiento con bevacizumab y corticoides, a pesar de lo cual, la evolución de la paciente fue tórpida y rápidamente fulminante llevándola a la muerte en menos de seis meses. La discusión que surge con este caso clínico, es si el tratamiento con OHB debería ser más enfocado a la prevención y profilaxis que al tratamiento de lesiones que por su ubicación anatómica revisten una alta gravedad y pueden ser irreversibles, una vez que se encuentran instauradas.
\end{abstract}

Palabras Clave: Oxygenation, hyperbaric, radiotherapy, medulloblastoma, radionecrosis, brain stem, HBO, CNS, HBOT, ATA, monoplace chamber, neuro-radionecrosis. 


\section{Introducción}

La radioterapia es uno de los tratamientos para el cáncer que más vidas ha salvado en los últimos años y adicionalmente los avances que se han dado en este campo, como los colimadores multi-hoja y los equipos de radioterapia 4D entre otros, han hecho que su eficiencia y eficacia se haya incrementado notablemente. Sin embargo, el riesgo de una radionecrosis siempre está latente y cuando el tejido involucrado es el tallo cerebral las consecuencias pueden incluso llegar a poner en peligro la vida del paciente, pues allí se encuentran los centros encargados del control neurovegetativo de funciones orgánicas que dan soporte vital, como son la respiración y el control de la frecuencia cardiaca y de múltiples funciones motoras y sensitivas, que tiene injerencia en prácticamente todo el organismo.

Esta complicación de la radioterapia, afortunadamente no es muy frecuente, pero es bastante impredecible y su ventana de aparición puede estar marcada por unos cuantos meses luego de la última irradiación hasta décadas, lo que hace todavía más complicado su seguimiento y diagnóstico.

Para el tratamiento de esta patología los expertos han utilizado diversos esquemas terapéuticos, que van desde el tradicional corticoide, hasta medicamentos de última generación como el bevacizumab que han mostrado una efectividad importante y de lo cual no nos ocuparemos en este artículo. Por otra parte, el OHB ha demostrado ser un excelente coadyuvante en el tratamiento de la radionecrosis hasta el punto de que la FDA ya ha aceptado su indicación en el caso de la radionecrosis ósea y de tejidos blandos.

No obstante, en lo concerniente a la aplicación del $\mathrm{OHB}$ en neuro-radionecrosis (NRN), aún hacen falta más estudios y sobretodo de incidencia estadística representativa, lo que no ha sido nada fácil considerando la baja frecuencia de esta complicación de la radioterapia en el sistema nervioso central (SNC). A pesar de lo anterior, los estudios existentes hasta el momento sugieren claramente que el oxígeno hiperbárico puede ser una herramienta importante como coadyuvante en el tratamiento de la NRN.

Desde el punto de vista fisiopatológico, el OHB basa su efectividad en 4 efectos demostrados, que pueden ser de utilidad para el tratamiento de la NRN:
1. Estimula la angiogénesis.

2. Mejora la oxigenación del tejido irradiado.

3. Disminuye la neuro-inflamación.

4. Disminuye la fibrosis.

Los mecanismos precisos para diferenciar los tipos de lesiones del SNC inducidos por la radiación no son del todo claros, los datos experimentales consistentes muestran un patrón de desarrollo de lesión que depende de dosis y tiempo.

Después de la radiación hay cambios severos en la barrera hemato-encefálica, que ocurren en tiempos diferentes después de la exposición, hay una disrupción temprana de la barrera y otra tardía. Los cambios reportados más consistentes son en el período agudo dependientes de la dosis y el incremento de la permeabilidad de la barrera la cual se recupera en pocas semanas. El desarrollo de la radionecrosis depende de la dosis/tiempo. Afecta los oligodendrocitos y las células endoteliales en forma directa y primaria, y los astrocitos se involucran en forma más secundaria, $y$ las neuronas se involucran en forma indirecta y en forma más tardía. La radionecrosis altera la barrera hemato-encefálica en forma temprana y luego en forma tardía. La integridad de la barrera depende de unión apretada de las células endoteliales y también de los pericitos y astrocitos que la rodea. La integridad de esta barrera es básica para la homeostasis.

La finalidad de este reporte de caso clínico es mostrar nuestra experiencia en el manejo coadyuvante con OHB en la NRN, pues lo que pudimos observar es que cuando se presenta esta complicación con un comportamiento tan mórbido y fulminante marcado principalmente por su ubicación en el tallo cerebral, no se logró detener el proceso, ni siquiera hacerlo más lento; pues por positivos que sean los efectos del OHB, es imposible revivir neuronas muertas o en proceso de muerte indefectible. Por todo lo anterior esperamos plantear adecuadamente la pregunta: ¿no habría sido mejor prevenir, que intentar evitar lo inevitable?, pregunta a la que daremos desarrollo más detenido en la Discusión al final del presente artículo.

\section{Descripción del caso clínico}

Se trata de una paciente de sexo femenino de 28 años de edad con historia de méduloblastoma que fue tratado con resección quirúrgica óptima en julio 
de 2009, quedándole un residuo tumoral inferior a 1.5 $\mathrm{cm}^{3}$. La patología tumoral reportó una lesión positiva para sinaptofisina (glicoproteína que se une al calcio que se encuentra en las membranas de las vesículas sinápticas) y CD 56 (marcadores para células Natural Killer), con lo que se confirmó actividad tumoral residual, con proliferación cuantificada por MIBI del 50 \% y con alguna diferenciación neuroblástica. Posteriormente y como tratamiento complementario se le realizó quimioterapia con cisplatino, etopósido, y ciclofosfamida, completando con radioterapia cráneo-espinal que se completó en octubre de 2009 con un total de $36 \mathrm{~Gy}$. En mayo del 2011 se le practicó una resonancia de control cuando la paciente estaba asintomática, se apreció muy leve aumento del volumen del tallo cerebral. En julio de 2011 presenta bradilalia, disartria, lateropulsión izquierda, diplopía, y cefalea occipital pulsátil de intensidad 8/10. Se realizó RMN GD que muestra cambios en el hemisferio cerebeloso derecho que sugieren una encefalomalacia, aumento considerable del diámetro del tallo cerebral en la protuberancia y el mesencéfalo, con alteración en la intensidad de la señal, cambio que se extiende hacia la región talámica y hacia el brazo posterior de la cápsula interna del lado derecho, y en menor grado hacia el lado izquierdo. Estos hallazgos en conjunto con la evidencia de un elevado pico de lactato parecen compatibles con radionecrosis, se inicio esquema antiedema cerebral y ciclo con bevacizumab y dexametasona en bolos, posteriormente presento marcha atáxica y espasticidad asimétrica con predominio en el hemicuerpo izquierdo, limitación para la deglución e incontinencia urinaria., Durante la exploración de los metabolitos, también se documentó un ligero aumento del mioinositol, hallazgo que hizo sospechar como segunda opción la presencia de una neoplasia meta- crómica no secundaria de bajo grado, evento que solo podría confirmarse con la realización de una biopsia como evento paralelo, o practicando un PET -11- MET. Después de discutir el caso con los grupos de neurocirugía, neuroradiología y oncología, se consideró continuar el tratamiento con la administración de Bevacizumab, anticipando la segunda serie tras completar 15 días de la primera dosis, y aumentando la dosis a $10 \mathrm{mg} / \mathrm{kilo}$. Además del registro periódico actual, continuo con bajas dosis de enoxaparina, y se le adicionó celecoxib, $200 \mathrm{mg} /$ día. Se dejó abierta la posibilidad de realizar terapia escalonada con Oxígeno Hiperbárico, como coadyuvante para contener la progresión del daño cito-tóxico instaurado por la teleterapia. Una espectroscopia por resonancia magnética, de septiembre 6 de 2011, reporta que los hallazgos son indicativos de cambios post-radioterapia, sin evidencia de lesión neoplásica activa.

\section{K.M.D \\ Radionecrosis por radioterapia}
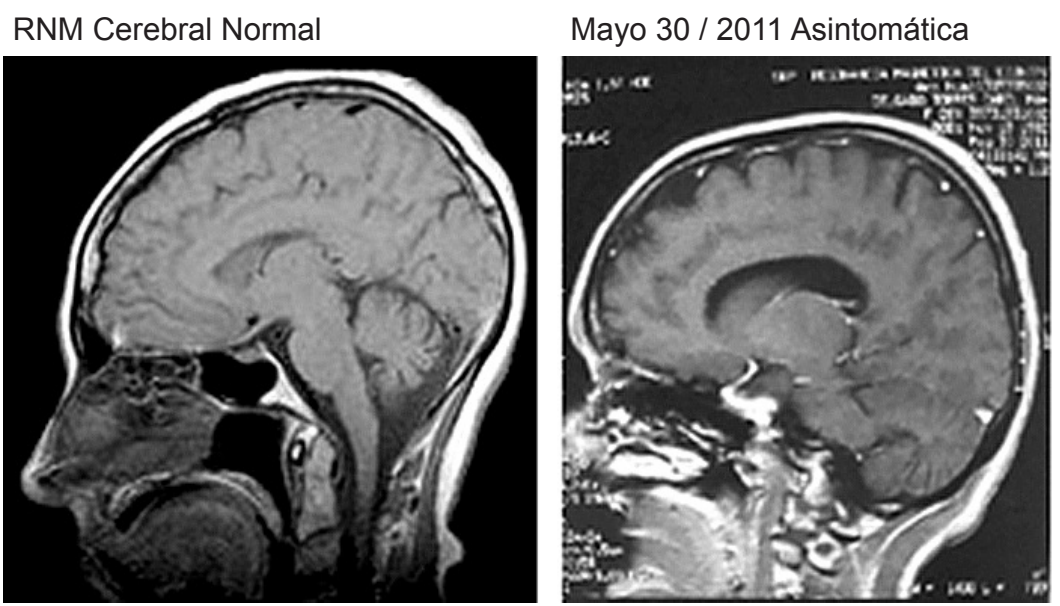

2 años después de la cirugía y radioterapia
Septiembre 6 / 2011 Sintomática

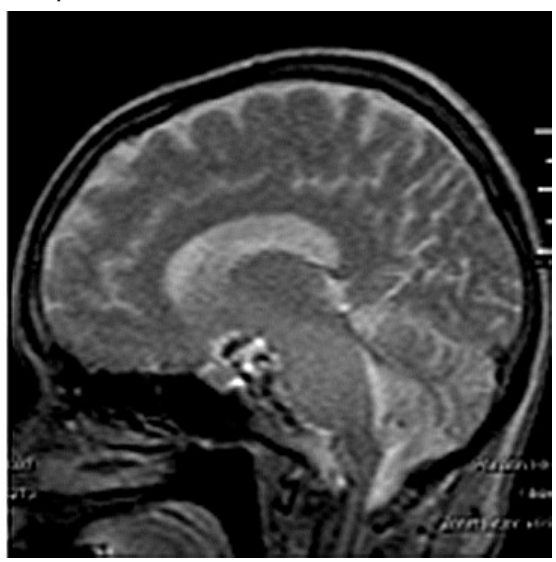

Espectroscopia por resonancia. Sintomática Dx Radionecrosis sin recidiva tumoral

Con la imagen de la derecha obtenida con espectroscopiía con resonancia se confirmó que la lesión presentada por la paciente se trataba de radionecrosis y no de una recidiva tumoral. 


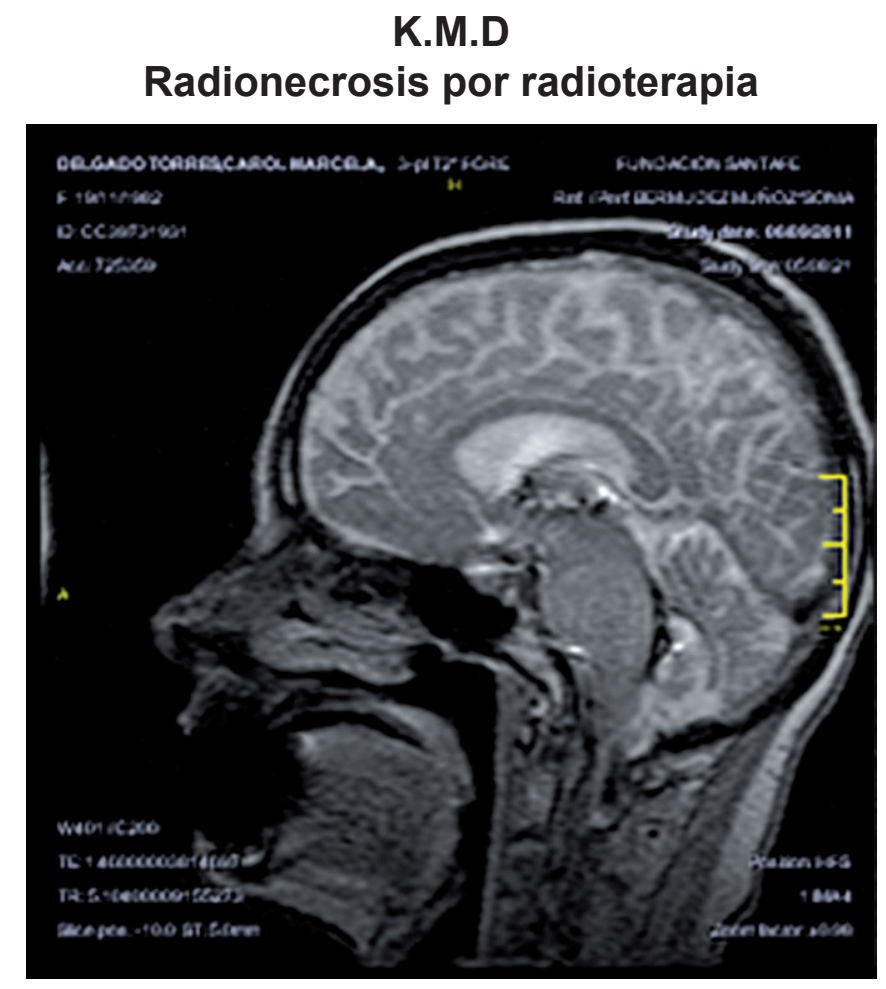

En esta imagen se observa el tallo cerebral muy engrosado secundario a una neuroinflamación por radioterapia.

\section{Radionecrosis 2 años después K.M.D}
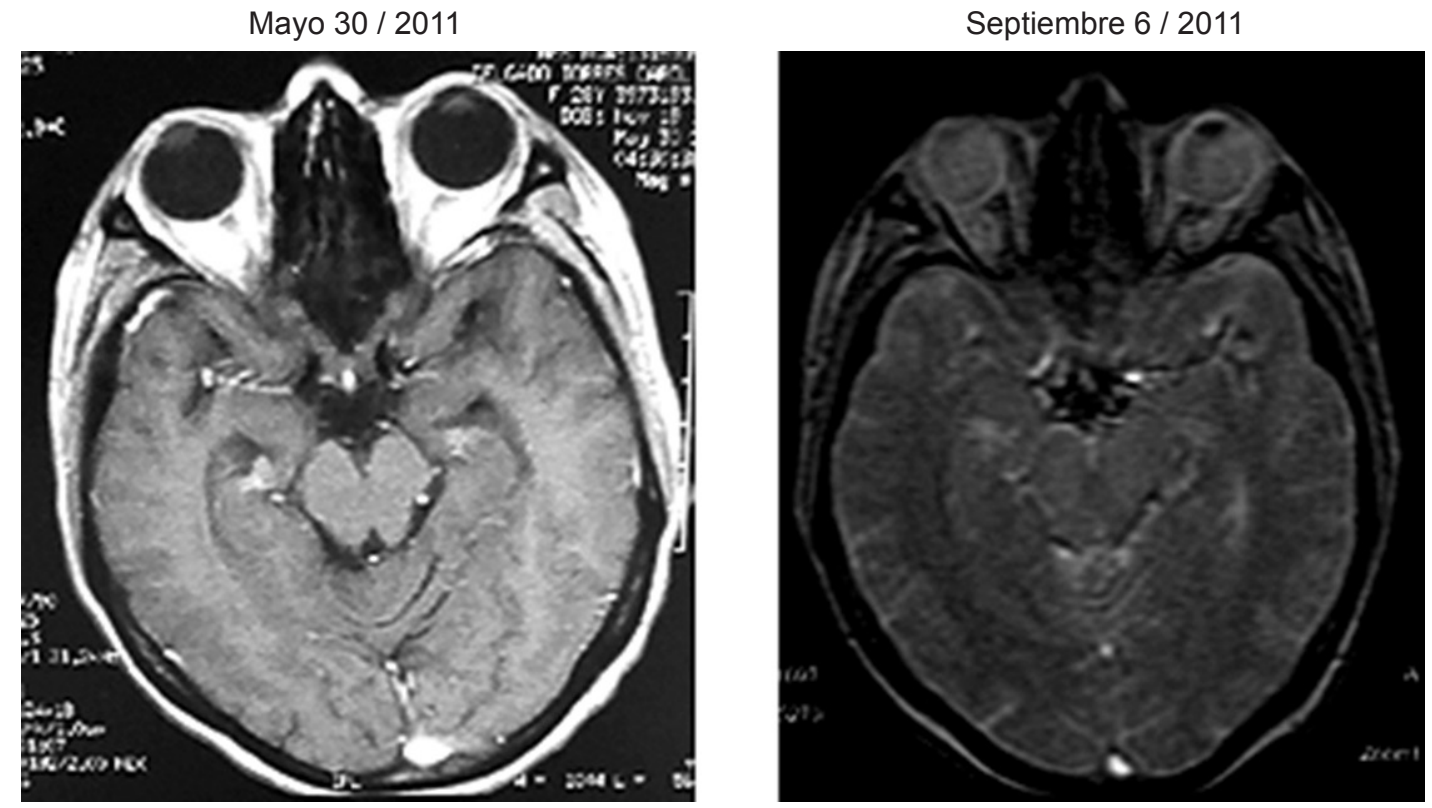

En esta imagen se observa a la izquierda una imagen de la paciente en mayo 30, completamente normal y en la imagen de la derecha tomada en septiembre del mismo año, se observa neuroinflamación, con borramiento de las cisternas basales y engrosamiento del pedúnculo cerebral izquierdo. 
Antecedentes de mamo plastia y abdominoplastia con fines estéticos. Al examen físico de ingreso se encontró paciente consciente, alerta, orientada, signos vitales estables y dentro de parámetros de normalidad. Al examen neurológico simetría facial conservada, disartria, fuerza en hemicuerpo izquierdo $+++/+++++$, rigidez espástica de pierna y pie izquierdos. Hiper-reflexia generalizada de predominio patelar bilateral. Control parcial de esfínteres.

Se realizaron 6 sesiones con oxígeno hiperbárico al $100 \%$ y a 1,8 ATA, cada una por 60 minutos en cámara monoplaza, los acompañantes (papá y hermana) informaron observar mejoría en la deglución y se hicieron menos frecuentes los episodios de microbroncoaspiración.

A pesar del tratamiento la paciente evoluciona tórpidamente, se incrementa la dificultad para la deglución por lo que se hace necesario realizar gastrostomía y rápidamente empeora su estado general.

El deterioro de la motilidad es rápido y severo y en menos de dos semanas pasa a una cuadriplejía casi completa, dificultad respiratoria por manejo inadecuado de las secreciones, imposibilidad para la deglución y afonía total. El estado de consciencia también empeora por somnolencia permanente y progresiva, que evoluciona llevándola a ser difícil de alertar, aunque permanece consciente y aparentemente con facultades mentales indemnes.

Ante el deterioro severo de la paciente, los familiares deciden interrumpir el tratamiento y pedir salida voluntaria para llevarla a la casa en donde finalmente fallece en noviembre de 2011.

\section{Discusión y conclusiones}

Cuando se inició el tratamiento con oxígeno hiperbárico en esta paciente, ya existía un diagnóstico clínico y para clínico confirmatorio de un proceso de radionecrosis avanzada post-radioterapia y sin recidiva tumoral. Es decir, que la OTHB se inició tres meses después de la aparición de los primeros síntomas y cuando la radionecrosis se encontraba en curso avanzado.

Aunque los familiares relataron alguna mejoría en la función deglutoria, lo cierto es que su evolución fue fulminantemente tórpida, por lo que al menos en este caso no se observó ninguna mejoría.
La conclusión que puede sacarse de este caso en particular es que una vez iniciado el proceso de radionecrosis tardía en el tallo cerebral, el proceso no puede ser detenido o al menos puede decirse que el oxígeno hiperbárico fue de poca utilidad en este caso concreto.

La discusión que planteamos en este caso es que el tratamiento con oxígeno hiperbárico debería aplicarse de manera profiláctica y no como tratamiento de una entidad plenamente establecida y cuyo curso rara vez es benévolo y menos cuando se encuentra ubicada en una zona tan vital como el tallo cerebral.

Si bien es cierto y está plenamente demostrado, que el oxígeno hiperbárico reduce los procesos de fibrosis, aumenta la vascularidad, facilita la hiperoxigenación de los tejidos e incrementa las células madre circulantes, efectos estos que podrían ser determinantes para evitar un proceso de radionecrosis, también es de aceptar, que la OTHB no puede lograr milagros como lo sería revivir células muertas.

Cuando se toma la decisión terapéutica de irradiar una zona tan delicada como el tallo cerebral y se asume la posibilidad de un desenlace fatal para el paciente, como un riesgo aceptable, definitivamente es justificable tomar todas las precauciones posibles, como lo sería el manejo profiláctico con oxígeno hiperbárico, iniciado 3 meses después de concluida la radioterapia.

\section{Referencias}

1. Regulations F. [Acceso 26 de septiembre de 2012]. FDA. Disponible en: www.accessdata.fda.gov/cdrh_docs/pdf8/k082455. pdf

2. Sheffield P. Aplication of Hyperbaric Oxygen Therapy in case of prolonged cerebral hypoxia following rapid decompresion. Aviat Space Environ Med. 1976; 47: 759-62.

3. Neubauer R. Cerebral oxygenation and the recoverable brain. Neurol Res. 1998. 20: s33-6.

4. Rossignol D. Hyperbaric oxygen therapy migth improve certain pathophysiological findings in autism. Medical Hypotheses. 2006; 2-20.

5. Soussain C. CNS complications of radiotherapy and chemotherapy. Lancet. 2009; 374: 1639-51.

6. Feldmeier, J. A systematic review of literature reporting the apllication of hyperbaric oxygen prevention and treatment of delayed radiation injuries: an evidence based approach. Undersea Hyperb Med., 1998; 29(1): 4-30. 